\title{
Membrana de fibrina versus injerto conectivo como tratamiento de recesión gingival
}

\section{Fibrin membrane versus connective tissue graft as gingival recession treatment}

\begin{abstract}
Andrés Gómez ${ }^{1 *}$, Ricardo Elgueta² ${ }^{2}$ Carola Ibieta ${ }^{1}$
1. Cirujano dentista, Especialista en Periodoncia, Profesor Universidad del Desarrollo, Concepción, Chile

2. Alumno post-título especialidad en Periodoncia, Universidad Andrés Bello, Concepción, Chile

Correspondencia a: Dr.andresgomez@gmail.com Trabajo recibido el 22/03/2015. Aprobado para su publicación el 12/07/2015

RESUMEN

Este caso clínico de boca dividida a 6 meses tiene como objetivo comparar los cados obtenidos con una membrana de fibrina rica en plaquetas y leucocitos (L-PRF) y un injerto de tejido conectivo (ITC) en el tratamiento de recesiones gingivales de Miller en un paciente con biotipo gingival grueso. El resultado muestra que el uso de una membrana de L-PRF provee un parcial cubrimiento de la recesión y un menor malestar subjetivo del paciente cuando se compara con el ITC. Sin embargo,

PALABRAS CLAVE

Rev. Clin. Periodoncia Implantol. Rehabil. Oral Vol. 10(1); 17-19, 2017.

ABSTRACT

The aim of this split-mouth clinical study, covering a 6 month period, is to compare the results obtained from Leucocyte and Platelet Rich Fibrin Membrane (L-PRF) and Connective Tissue Grafting (CTG) in the treatment of class 1 Miller gingival recessions in a patient with thick gingival biotype. The results show that the use of L-PRF membrane provides partial coverage of the recession, as well as lower subjective patient discomfort when compared with CTG. However, CTG provides a higher percentage of 6 months.

KEYWORDS

Connective tissue graft; Fibrin membrane; Recession; Mucogingival surgery

Rev. Clin. Periodoncia Implantol. Rehabil. Oral Vol. 10(1); 17-19, 2017.
\end{abstract}

\section{INTRODUCCIÓN}

La recesión gingival es definida como el desplazamiento del margen gingival apical al límite amelocementario (LAC) ${ }^{1,2,3}$ (fig.1). Obtener un predecible cubrimiento radicular con un óptimo nivel de estética es una realidad en cirugía plástica periodontal ${ }^{4}$. La membrana de fibrina rica en plaquetas y leucocitos (L-PRF) corresponde a una nueva generación de concentrados plaquetarios con un procedimiento simplificado. Fue desarrollada en Francia por Choukroun et al. ${ }^{4,5}$, y su método simplificado no requiere la aplicación de anticoagulante. Su estructura tridimensional contiene plaquetas, leucocitos, citoquinas, factores de crecimiento y células mesenquimáticas indiferenciadas. Muestra un efecto promotor de la neoangiogénesis ${ }^{5}$ y puede ser usada como biomaterial de cicatrización $n^{4-8}$.

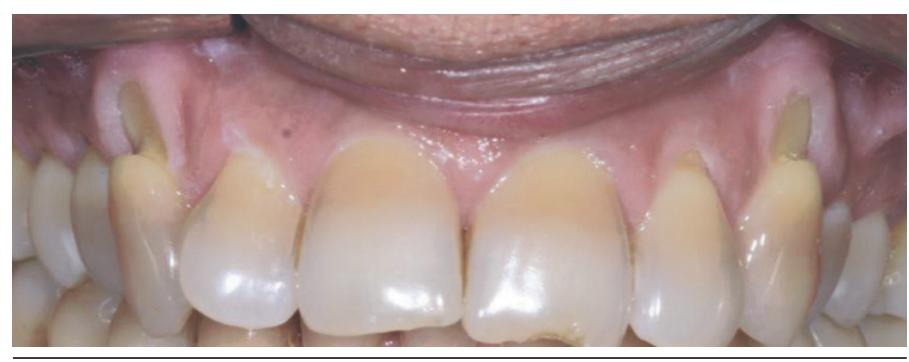

Figura 1. Recesiones clase 1 de Miller, simétricas bilaterales

\section{MATERIALES Y MÉTODOS}

Fue seleccionada una paciente de género femenino, de 44 años de edad, sistémica y periodontalmente sana, sin hábito tabáquico, con recesiones simétricas por abfracción y cepillado traumático, bilaterales en dientes 1.3 y 2.3 vitales y libres de restauraciones. Posteriormente fue instruida con una técnica de cepillado y elementos de higiene en conjunto a un tratamiento periodontal adecuado. Luego fue informada de las características del estudio, dando su consentimiento informado por escrito. El trabajo cumple con la declaración de Helsinki. Posteriormente, un examinador ciego y calibrado, previo al tratamiento quirúrgico y 6 meses posteriores a él, recolectó los siguientes datos en ambos grupos:

A) Características clínicas visuales en relación a la recesión. B) Medición del biotipo gingival mediante el método de la traslucidez (fig. 2). C) Medición de la recesión gingival. D) Evaluación RES (Root coverage Esthetic Score, sistema propuesto para evaluar el resultado estético de los procedimientos de cobertura radicular) a los 6 meses.

Todas las mediciones fueron realizadas por el mismo operador. La asignación del grupo quirúrgico test, control y el sitio quirúrgico inicial fue realizada al azar. Ambas recesiones fueron tratadas en el mismo acto quirúrgico. En un lado se realizó un colgajo desplazado coronal más injerto de tejido conectivo ( $C D C+I T C)$ y en el otro un colgajo desplazado coronal más una membrana de fibrina rica en plaquetas y leucocitos (CDC + L-PRF). 


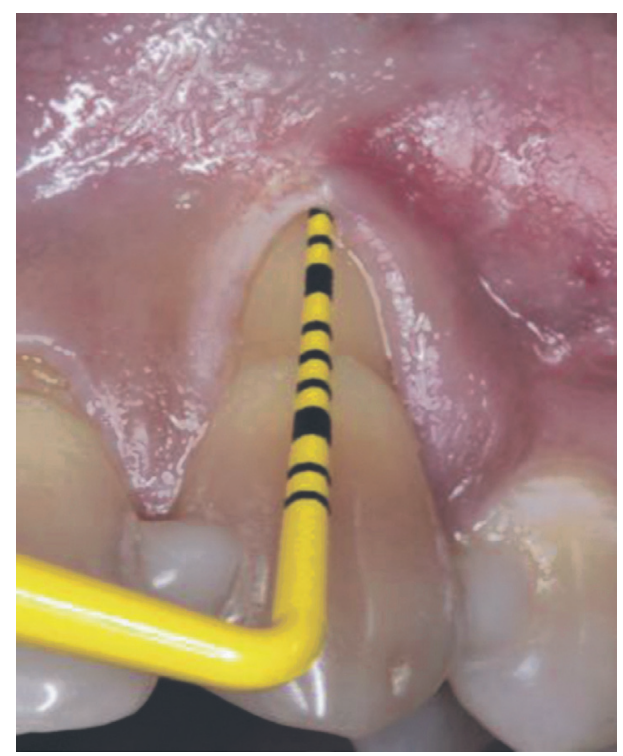

Figura 2. Evaluación de biotipo gingival con sonda periodontal (Colorvue, Hu- Friedy®), biotipo grueso.

\section{Protocolo L-PRF y quirúrgico}

Mediante una punción venosa al vacío con mariposa $\left(n .^{\circ} 23 \mathrm{G}\right.$, «Blood Collection Set+Luer Adapter» [0,64×19mm], Vacuette ${ }^{\circledR}$ ) y camisa plástica (BD Vacutainer $\left.{ }^{\circledR}\right)$ se dispensaron muestras de sangre en 6 tubos de $9 \mathrm{ml}$ cada uno (Vacuette $®$ ), las cuales fueron centrifugadas (Tomos Low Speed Centrifuge model 1-4 ${ }^{\text {a }}$, Intop Biolab INC $($ ) inmediatamente a $2.700 \mathrm{rpm}$ durante $12 \mathrm{~min}$.

Después de haber anestesiado con articaína 4\% (Septanest $囚$ ), las superficies radiculares fueron pulidas con una cureta tipo Gracey Minifive 1-2 (Hu-Friedy $\left.{ }^{8}\right)$ y posteriormente acondicionadas con una solución de tetraciclina ${ }^{9}$ ( 250 mg diluidos en $1 \mathrm{cc}$ de suero, tetraciclina L.CH ${ }^{\circledR}$ ) por $1 \mathrm{~min}$. Se realizaron incisiones intrasurculares, a modo de bolsillo ${ }^{10}$, en la zona de $\mathrm{p} 1.3$ y $2.3^{10}$. Un colgajo de espesor parcial a capa superficial fue levantado más allá de la línea mucogingival partiendo desde el margen de la recesión.

Las 6 membranas de L-PRF y el injerto de tejido conectivo fueron puestos en los respectivos sitios receptores; las membranas de L-PRF fueron puestas una sobre otra y en direcciones opuestas ${ }^{7}$ (figs. 3a-b y 4 c figs. 3 y 4). La capa superficial del colgajo fue desplazada $2 \mathrm{~mm}$ coronal al LAC y fijada mediante una sutura colchonero vertical anclada a un punto de contacto creado artificialmente con composite (Flow z350, $3 \mathrm{M} \otimes)$ en proximal de los sitios en estudio ${ }^{7}$. Luego, para aumentar más la estabilidad, se realizó una sutura colchonera horizontal cruzada con anclaje a palatino de la pieza dentaria. Las suturas empleadas fueron de tipo reabsorbibles $5 / 0$ (Safil囚). La técnica de obtención del injerto conectivo se realizó mediante una incisión horizontal paralela a las piezas dentarias ubicadas entre 1.7 y $1.3 ; 3 \mathrm{~mm}$ apical al margen gingival y perpendicular a este, hasta sentir contacto óseo, con una pequeña incisión distal perpendicular a la primera. Luego se realizó una disección de $1,5 \mathrm{~mm}$ de espesor hacia la línea media palatina para posteriormente trazar con un bisturí todo el perímetro del injerto, que se despegará mientras es prendido con una pinza recta. Luego de retirado el injerto,
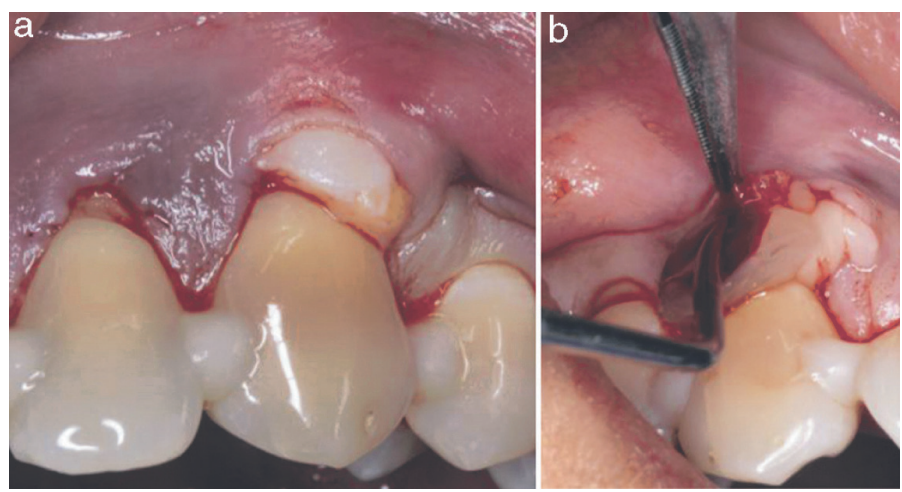

Figura 3. a) Interposición de injerto de tejido conectivo. b) Membranas de L-PRF. Nótense los puntos de contacto creados para anudar y desplazar a incisal el colgajo, mejorando los vectores de fuerza.
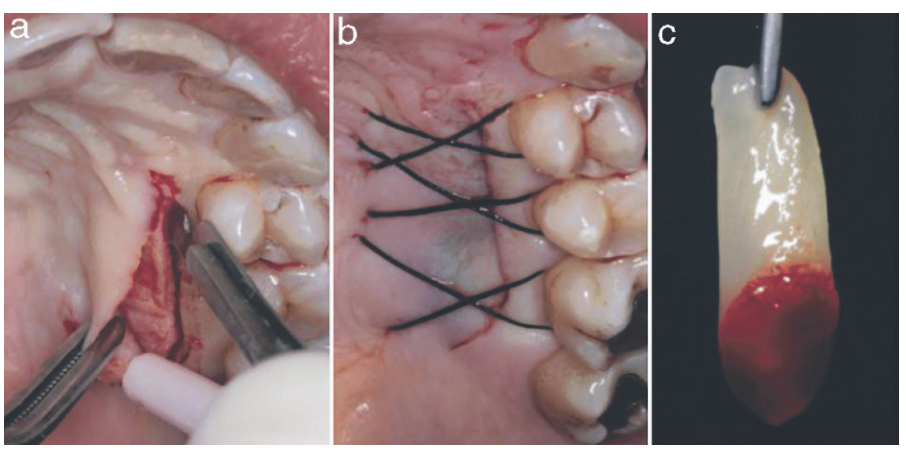

Figura 4. a,b) Técnica de obtención de injerto de tejido conectivo y posterior sutura. c) Membrana de L-PRF. Nótese su área roja o cabeza, zona rica en factores de crecimiento.

el paladar se suturó con nudos colchoneros horizontales cruzados con anclaje dentario vestibular. El material usado fue seda $3 / 0$ (Ethicon®) (fig. $4 \mathrm{a}, \mathrm{b})$.

El paciente fue mantenido con un colutorio de clorhexidina al $0,12 \%$ (Perioaid@) 2 veces al día durante 21 días, además de seguir indicaciones dadas por escrito referentes a cirugías mucogingivales ${ }^{7,11-13}$.

\section{RESULTADOS}

La tabla 1 muestra el resultado de las evaluaciones de ambos sitios quirúrgicos al día 0 o día de la cirugía y su control a los 6 meses (fig. 5a-f). En ambos sitios intervenidos hubo una disminución de la recesión, pero
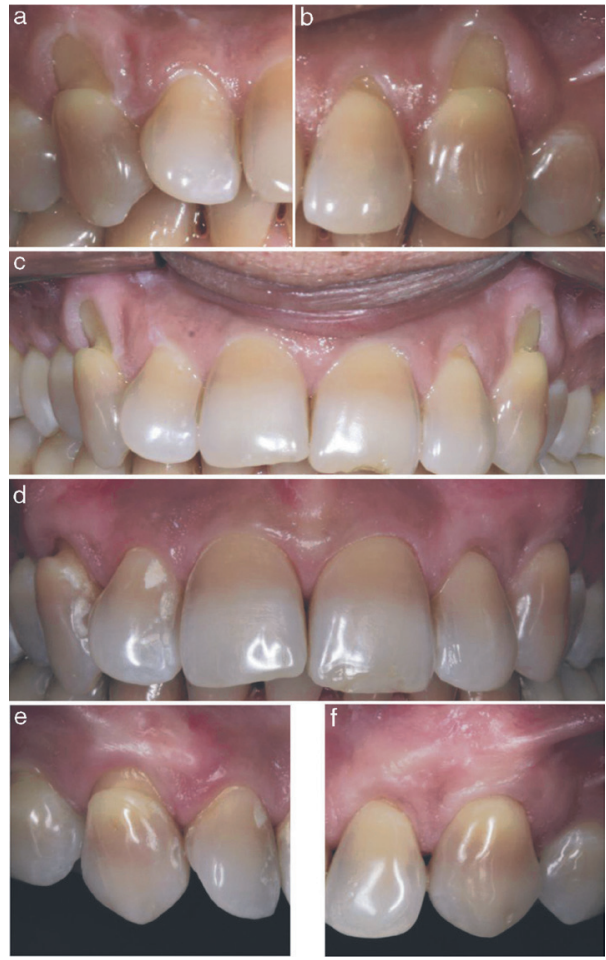

Figura 5. Fotografías comparativas, iniciales y a los 6 meses. Izquierda: p 1.3 (CDC+L-PRF). Derecha: p 2.3 (CDC+ ITC). a) p1.3 día 0; b) p2.3 día 0 ; c) frontal día 0 ; d) frontal 6 meses poscirugía; e) p1.3, control 6 meses; f) p2.3, control 6 meses.

este cubrimiento radicular fue mayor en el diente donde se usó el ITC. El resultado estético, evaluado en la tabla 2 mediante el Red Esthetic Score (RES) ${ }^{3-12}$, también tuvo un mejor resultado en la zona donde se usó el ITC. La diferencia a favor de este se determinó al evaluar la posición del margen gingival, donde obtuvo un cubrimiento completo. Color y contorno del margen gingival, textura de los tejidos blandos, alineación de la unión mucogingival y color de la encía obtuvieron resultados similares con ambas técnicas. 
Tabla 1. Medición de la recesión gingival en milímetros, al ingreso del paciente y a los 6 meses poscirugía

\begin{tabular}{|c|c|c|c|c|c|c|c|c|}
\hline \multirow[t]{2}{*}{ Diente } & \multicolumn{2}{|c|}{$\begin{array}{l}\text { [0,2-3]Ancho } \\
\text { M-D zona } \\
\text { recesión }\end{array}$} & \multicolumn{2}{|c|}{$\begin{array}{l}\text { [0,4-5]Largo } \\
\text { LAC-Margen } \\
\text { gingival }\end{array}$} & \multicolumn{2}{|c|}{$\begin{array}{l}\text { [0,6-7]Prof. } \\
\text { sondaje } \\
\text { Margen-fondo } \\
\text { surco }\end{array}$} & \multicolumn{2}{|c|}{$\begin{array}{l}{[0,8-9] N . I . C .} \\
\text { LAC-fondo } \\
\text { surco }\end{array}$} \\
\hline & $\begin{array}{c}\text { Día } \\
0\end{array}$ & $\begin{array}{c}6 \\
\text { meses }\end{array}$ & $\begin{array}{c}\text { Día } \\
0\end{array}$ & $\begin{array}{c}6 \\
\text { meses }\end{array}$ & $\begin{array}{c}\text { Día } \\
0\end{array}$ & $\begin{array}{c}6 \\
\text { meses }\end{array}$ & $\begin{array}{c}\text { Día } \\
0\end{array}$ & $\begin{array}{c}6 \\
\text { meses }\end{array}$ \\
\hline 1.3 & 4 & 3 & 4 & 2 & 3 & 1 & 7 & 3 \\
\hline 2.3 & 4 & 0 & 4 & 0 & 2 & 1 & 6 & 1 \\
\hline
\end{tabular}

\section{DISCUSIÓN}

Las recesiones gingivales son un problema funcional-estético recurrente y de especial atención en lo que corresponde a la cirugía plástica periodontal. La mayoría de los tratamientos en las recesiones gingivales dan resultados predecibles en recesiones clase I y II de Miller con el uso de CDC por sí solo o con el uso de injertos. La literatura también reporta una amplia variación para el parámetro clínico de cubrimiento radicular, con rangos que van entre el 70 al $98 \%$ 1,2,3,8,12,14,15. Es aquí donde la membrana de fibrina podría ser una opción interesante como injerto, debido a la gran cantidad de factores de crecimiento, que cumplirían un rol crucial en la regeneración tisular $6,8,14,13$.

El uso de L-PRF en el tratamiento de recesiones gingivales en adición a un colgajo desplazado a coronal demostró tempranamente una mejoría durante la fase de cicatrización, obteniendo un buen remodelado en grosor y estabilidad, así como también en el bienestar postoperatorio y aceptación de tratamiento por parte del paciente. Todo esto además de la ventaja de no tener que invadir la zona del paladar, como zona donante. Sin embargo, a los 6 meses se comprueba que la cobertura radicular con membrana de L-PRF+CDC es menor en comparación al uso de ITC+CDC. Quizás el método de recolección de sangre y el posterior ciclo de centrifugado pudieron haber generado a nivel estructural un tipo de membrana no óptimo para una cirugía de cubrimiento radicular. Además, en este estudio la restitución del LAC mediante composite pudo haber influido en la valoración de la recesión y en su profundidad. La profundidad de la recesión, o abfracción, pudo ser un parámetro determinante en el porcentaje de cubrimiento de la recesión, el cual no fue evaluado y podría haber condicionado el resultado.

Existe en ambas terapias una disminución de la recesión (ancho y largo) y ganancia de inserción clínica, así como también una similar disminución en la profundidad de sondaje, resultando en un aumento de espesor de tejido queratinizado, lo que podría significar obtener a largo plazo resultados clínicos estables y una menor probabilidad de recesión futura.

Se establece además un análisis estético (RES) cuyos valores en el uso de L-PRF (RES: 7) demostraron una diferencia de 3 puntos con
Tabla 2. Evaluación Red Esthetic Score (RES) 6 meses poscirugía

\begin{tabular}{lcc}
\hline \multicolumn{1}{c}{ PARÁMETRO A EVALUAR } & $\mathbf{1 . 3}$ & $\mathbf{2 . 3}$ \\
\hline Posición del margen gingival (0-3-6) & 3 & 6 \\
Contorno del margen gingival (0-1) & 1 & 1 \\
Textura de los tejidos blandos (0-1) & 1 & 1 \\
Alineación de la unión mucogingival (0-1) & 1 & 1 \\
Color de la encía (0-1) & 1 & 1 \\
Total & 7 & 10
\end{tabular}

respecto al uso de ITC (RES: 10). La diferencia obtenida fue por existir en el injerto de L-PRF una posición del margen gingival apical al LAC, ya que la recesión no fue cubierta del todo. Esto podría explicarse por la técnica de desplazamiento coronal empleada ${ }^{10}$. La que por el contrario, al no tener descargas, permite un resultado estético más predecible que aquellas que sí la tienen. Podría ser posible que, quizás, con una técnica que favorezca más el desplazamiento coronal y un mejor acceso visual para la colocación superpuesta y en direcciones opuestas de las membranas de fibrina, como una técnica de Langer, la cobertura hubiese sido mayor ${ }^{12}$.

\section{CONCLUSIÓN}

El presente caso clínico muestra que el uso de CDC+ITC en una recesión gingival tipo I de Miller resulta ser un tratamiento predecible y de primera elección para la cobertura radicular tanto en función como estética ${ }^{1,2,3,11,12,14,15}$, si se compara con un CDC+L-PRF. El uso de $C D C+L-P R F$ es una técnica que muestra la ventaja de un menor dolor postoperatorio ${ }^{7}$, se evita la intervención de la zona donante y se obtienen valores clínicos aceptables, pero los resultados en nuestro caso clínico no fueron similares al sitio donde se usó un ITC.

Si bien hubo un resultado aceptable, son necesarios más estudios para evaluar usos clínicos de la membrana de L-PRF en el tratamiento de cobertura radicular en recesiones gingivales.

\section{CONFLICTO DE INTERESES}

Los autores declaran no tener conflicto de intereses.

\section{AGRADECIMIENTOS}

Agradecimiento especial a la Universidad del Desarrollo, Sede Concepción, por facilitar su infraestructura para el desarrollo de este trabajo, y a mis colegas Dr. Cristian López, Roxana Vargas y Ángela Suazo por su contribución en la investigación y preparación del manuscrito.

\section{Bibliografía}

1. Baldi C, Pini-Prato G, Pagliaro U, Nieri M, Saletta D, Muzzi L,et al. Coronally advanced flap procedure for root coverage. Isflap thickness a relevant predictor to achieve root coverage? A19-case series. J Periodontol. 1999;70:1077-84.

2. Zucchelli G, de Sanctis M. Treatment of multiple recession-type defects in patients with esthetic demands. J Periodontol.2000;71:1506-14.

3. Cairo F, Rotundo R, Miller PD, Pini Prato GP. Root coverage est-hetic score: A system to evaluate the esthetic outcome of thetreatment of gingival recession through evaluation of clinicalcases. J Periodontol. 2009;80:705-10.

4. Choukroun J, Adda F, Schoeffer C, Vervelle APRF. An oppor-tunity in perioimplantology [en francés]. Implantodontie.2000;42:55-62.

5. Choukroun J, Diss A, Simonpieri A, Girard MO, Schoeffler C,Dohan SL, et al. Platelet-rich fibrin (PRF): A second-generationplatelet concentrate. Part IV: Clinical effects on tissue hea-ling. Oral Surg Oral Med Oral Pathol Oral Radiol Endod.2006;101:e56-60.

6. Jankovic $S$, Klokevold $P$, et al. Use of platelet-rich fibrinmembrane following treatment of gingival recession: A ran-domized clinical trial. Int $\mathrm{J}$ Periodontics Restorative Dent.2012;32:e41-50

7. Aroca S, Keglevich T, Barbieri B, Gera I, Etienne D. Clinicalevaluation of a modified coronally advanced flap alone or incombination with a platelet-rich fibrin membrane for the treat-ment of adjacent multiple gingival recessions: A 6-month study.J Periodontol. 2009;80:244-52.

8. Reddy S, Prasad MGS, Agnihotri J, Amudha D, Singh S, Krishna-nand P. Management of multiple recession defect using modifiedcoronally advanced flap alone or with PRF. Int J Health Sci.2013;3:133-8.
9. Trombelli L, Scabbia A, Wikesjo UM, Calura G. Fibrin glue appli-cation in conjunction with tetracycline root conditioning andcoronally positioned flap procedure in the treatment of humangingival recession defects. J Clin Periodontol. 1996;23:861-7

10. Raetzke Peter B. Covering localized areas of root expo-sure employing the envelope technique. J Periodontol.1985;56:397-402.

11. Graziani F, Gennai S, Roldán S, Discepoli N, Buti J, Madia-nos P, et al. Efficacy of periodontal plastic procedures in thetreatment of multiple gingival recessions. J Clin Periodontol.2014;41:S63-76.

12. Cairo F, Nieri M, Pagliaro U. Efficacy of periodontal plas-tic surgery procedures in the treatment of localized facialgingival recessions. A systematic review. J Clin Periodontol.2014;41:S44-62.

13. Alkan EA, Parlar A. EMD or subepithelial connective tissue graftfor the treatment of single gingival recessions: A pilot study. JPeriodontal Res. 2011;46:637-42.

14. Cortellini P, Pini Prato G. Coronally advanced flap and com-bination therapy for root coverage. Clinical strategies basedon scientific evidence and clinical experience. Periodontol.2012;2000:158-84.

15. Cortellini P, Tonetti M, Baldi C, Francetti L, Rasperini G, RotundoR, et al. Does placement of a connective tissue graft improvethe outcomes of coronally advanced flap for coverage of sin-gle gingival recessions in upper anterior teeth? A multicentre,randomized, double-blind, clinical trial. J Clin Periodontol.2009;36:68-79. 